\title{
High-precision VTEC derivation with GEONET
}

\author{
Qi Li ${ }^{1,2^{*}}$ (D, Guanyi Ma ${ }^{1,2}$, Takashi Maruyama ${ }^{1,3}$, Jinghua Li ${ }^{1}$, Qingtao Wan ${ }^{1}$, Xiaolan Wang ${ }^{1}$, Jiangtao Fan ${ }^{1}$ \\ and Jie Zhang'
}

\begin{abstract}
This paper proposes a new technique, namely Phase bias-based Small Grid Model (PSGM), to derive absolute ionospheric vertical total electron content (VTEC) with observations of Global Navigation Satellite System Earth Observation Network of Japan (GEONET). The proposed technique deals with the phase observations alone without handling the pseudoranges, which reduces the noise in VTEC estimation. A new parameter, the arc bias $\left(B_{\text {arc }}\right)$, is introduced to combine the phase ambiguities and differential phase biases. To solve $B_{\text {arc }}$ equations are constructed under the assumption that the VTEC is identical in the same $0.1^{\circ} \times 0.1^{\circ}$ grid. The performance of PSGM is evaluated with the observations in solar maximum year 2014. The root mean square error (RMSE) of PSGM is 0.40 TECU in average, the maximum RMSE is 0.73 TECU and the minimum RMSE is 0.26 TECU. The fitting accuracy of the VTEC results is improved compared with most of the existing methods.
\end{abstract}

Keywords: PSGM, TEC, lonosphere, GEONET, GPS, IRI, Mid-latitude

\section{Introduction}

In the past 30 years, dual-frequency Global Positioning System (GPS) has become the most important approach for absolute vertical total electron content (VTEC) measurement. The differential code bias (DCB) of GPS hardware device is an important factor affecting the VTEC estimation. To get absolute VTEC, scientists have proposed many methods to remove DCB (e.g., Mannucci et al. 1998; Sarma et al. 2008; Zhang et al. 2009). These methods are usually based on the assumption that the DCBs of each satellite/receiver remain unchanged for a period of time. The DCBs were assumed to remain unchanged over several hours ( $\mathrm{Li}$ et al. 2015), over an entire day (e.g., Arikan et al. 2008; Ma and Maruyama 2003; Li et al. 2018), over several days (Otsuka et al. 2002) or even over several months (Han et al. 2018).

Another basic assumption of the existing VTEC and DCB fitting models is that VTEC is smooth over a short period and a small region. The polynomial model was proposed in early times, which is suitable for local

\footnotetext{
*Correspondence: liqi@nao.cas.cn

${ }^{1}$ National Astronomical Observatories, Chinese Academy of Sciences,

Beijing, China

Full list of author information is available at the end of the article
}

region of single station (Lanyi and Roth 1988; Coco et al. 1991). The VTEC was assumed to conform to a polynomial function of longitude and latitude. Later under the assumption that VTEC is smooth over time, a Kalman filter model was proposed (Sardon et al. 1994). To fit the global ionosphere model, the VTEC was mapped to a spherical surface harmonic function of longitude and latitude with global GPS network (Wilson et al. 1995). A well trained multilayer neural network, which is equivalent to a nonlinear, smooth and interpolated function, was applied to VTEC and DCB estimation (Ma et al. 2005; Perez 2019). Assuming that VTEC is smoothed in a small area, DCBs were estimated by constructing convolution equation set with over 1300 Global Navigation Satellite System Earth Observation Network of Japan (GEONET) stations (Li et al. 2018).

The precision of absolute VTEC estimating is not only very important for scientific research, but also affects the accuracy of space applications such as satellite navigation and communications, and remote sensing. The efforts to obtain absolute VTEC with higher precision, smaller spatial-scale and shorter temporal-scale have never ceased. Using around 300 GPS stations from GEONET, and assuming that the VTEC is identical at any point within a $2^{\circ} \times 2^{\circ}$ grid, the VTEC and DCBs were determined by grid method 
(Ma and Maruyama 2003) with the standard deviation of satellite DCBs from 0.22 TECU to 1.89 TECU and the standard deviation of receiver DCBs smaller than 5.7 TECU. Zhang et al. (2010) estimated GPS DCBs with $0.5^{\circ} \times 0.1 \mathrm{~h}$ grids $\left(0.1 \mathrm{~h}\right.$ is equivalent to $1.5^{\circ}$ in longitude as earth rotation), and found the RMS of estimated DCBs from the data observed in the low latitude region is larger than middle latitude, the RMS value varies from approximately 1 to 3 TECU, but all RMS values are less than 4 TECU. DCBs obtained by single-station method and multi-station methods were compared by Choi et al. (2013), it is found that the accuracy of DCB fitted by multi-station method is better than that of single-station method. Receiver DCB was usually believed to be affected by temperature, but even after the temperature dependence is removed, a noise level of 1-3 TECU still remains in the DCB estimation (Coster et al. 2013). With Multi-GNSS Observations and Global Ionosphere Maps, DCB's day-to-day variation was estimated for satellites and receivers: DCB's day-to-day variation in satellite was usually smaller than $0.86 \mathrm{TECU}$ and in receiver smaller than 5.7 TECU (Montenbruck et al. 2014).

In this paper, in order to obtain more accurate absolute VTEC, a phase bias-based Small Grid Model (PSGM) is proposed for VTEC derivation with GEONET, which is the world's densest GNSS observation network. New assumptions are introduced as the basis of the model. Pseudorange measurements will not be used at all, but only GPS phase measurements are used for calculation as carrier phase measurement has higher precision. A new parameter $B_{\text {arc }}$ is introduced to represent hardware delay biases and integer ambiguities. Finally, the accuracy of PSGM are validated with the observation in solar maximum year 2014, and the estimated VTEC result is compared with the International Reference Ionosphere 2016 (IRI) model (Bilitza et al. 2017) and the VTEC products of University of Bern (UNIBE) (Schaer et al. 1996).

\section{Algorithms of PSGM}

Assuming that all the electrons in the ionosphere are concentrated on an infinitely thin spherical shell, then for a pair of receiver and satellite, the basic formulas for VTEC estimation with GPS phase observations are:

$$
\begin{aligned}
& \text { VTEC }=\operatorname{STEC} * \cos \chi, \\
& \mathrm{STEC}=n_{0}\left(L_{1} \lambda_{1}-L_{2} \lambda_{2}\right)+B_{\mathrm{arc}},
\end{aligned}
$$

where $X$ is the zenith of the optical path (Ma and Maruyama 2003). $\lambda_{1}, \lambda_{2}$ and $L_{1}, L_{2}$ represent wave lengths (unit: $\mathrm{m})$ and phases (unit: cycle) observations of the corresponding carriers. $n_{0}=\frac{2\left(f_{1} f_{2}\right)^{2}}{k\left(f_{1}^{2}-f_{2}^{2}\right)}=9.517 * 10^{16} \mathrm{~m}^{-3}$ is a constant, indicates phase delay difference of $1 \mathrm{~m}$ corresponding to the TEC of 9.517 TECU, where $f_{1}=1.57542 \mathrm{GHz}$ and $f_{2}=1.22760 \mathrm{GHz}$ are the frequencies of the two GPS carriers and $k=80.62 \mathrm{~m}^{3} / \mathrm{s}^{2}$ is a constant related to the charge and mass of electron (Ma and Maruyama 2003). STEC represents the optical path integration of electron density. In Formula (2), $B_{\text {arc }}$ contains contribution from integer ambiguities and differential phase biases of both carriers based on the hypothesis that all these parameters are invariant within the same phase connected arc (Blewitt 1990; Hernández-Pajares et al. 1999).

The ionospheric shell concerned is divided into small grids according to $0.1^{\circ}$ by $0.1^{\circ}$ in latitude and longitude, and the VTEC in the same grid at the same time is assumed identical. If the ionospheric piercing points IPP and $\mathrm{IPP}_{j}$ of two arcs $i, j$ are located in the same grid at time $t$, then there is:

$$
\operatorname{VTEC}_{i}^{t}-\mathrm{VTEC}_{j}^{t}=0 .
$$

By taking Formulas (1) and (2) into (3), an equation containing two unknowns, $B_{\text {arci }}$ and $B_{\text {arci }}$, can be obtained:

$$
B_{\mathrm{arci} i} \cos \chi_{i}^{t}-B_{\mathrm{arcj}} \cos \chi_{j}^{t}=\operatorname{sTEC}_{j}^{t} \cos \chi_{j}^{t}-\operatorname{sTEC}_{i}^{t} \cos \chi_{i}^{t} .
$$

Using all eligible pairs of IPPs in a day to build equations, these equations can form an overdetermined equation set:

$$
A * B=C,
$$

where

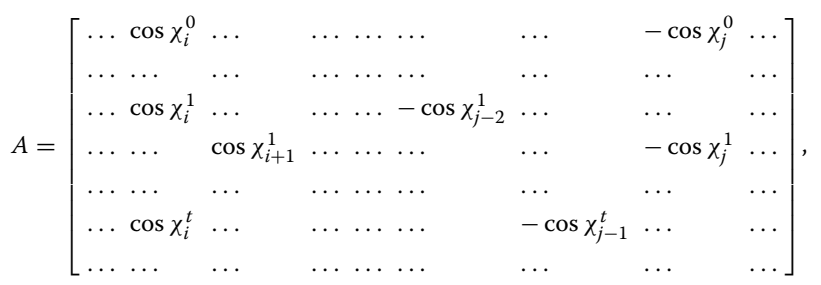

$$
B=\left[\begin{array}{r}
B_{\text {arc1 }} \\
\ldots \\
B_{\text {arci }} \\
\cdots \\
B_{\operatorname{arcj}} \\
\ldots \\
B_{\operatorname{arc} 40000} \\
\cdots
\end{array}\right], \quad C=\left[\begin{array}{r}
\operatorname{sTEC}_{j}^{0} \cos \chi_{j}^{0}-\operatorname{sTEC}_{i}^{0} \cos \chi_{i}^{0} \\
\ldots \\
\operatorname{sTEC}_{j-2}^{1} \cos \chi_{j-2}^{1}-\operatorname{sTEC}_{i}^{1} \cos \chi_{i}^{1} \\
\operatorname{sTEC}_{j}^{1} \cos \chi_{j}^{1}-\operatorname{sTEC}_{i+1}^{1} \cos \chi_{i+1}^{1} \\
\cdots \\
\operatorname{sTEC}_{j-1}^{t} \cos \chi_{j-1}^{t}-\operatorname{sTEC}_{i}^{t} \cos \chi_{i}^{t} \\
\cdots
\end{array}\right] .
$$

$B$ is solved by least square method:

$$
\widehat{B}=\left(A^{\mathrm{T}} A\right)^{-1} A^{\mathrm{T}} C .
$$

Then, the fitting residuals are: 


$$
D=A * \widehat{B}-C .
$$

The root mean square error (RMSE) of the fitting residuals is used to measure the accuracy of the model:

$$
R M S E=\sqrt{\frac{\sum_{n=1}^{L} \Delta_{n}^{2}}{L}},
$$

where $L$ represents the number of equations and $\Delta_{n}$ represents the residual of the $n$th equation.

The observation data are from over 1300 GEONET stations with $1 / 30 \mathrm{~Hz}$ sampling frequency of 364 days in 2014. With $30^{\circ}$ cutoff angle of satellite elevation, and after the arcs with cycle slip are removed, more than 40,000 arcs a day are generated. The number of equations per day is about $3 \times 10^{6}$. So $A$ is a sparse matrix of about 3,000,000 $\times 40,000$; the giant matrix Eq. (5) is solved by the high performance computing cluster of the Information and Computing Center of National Astronomical Observatories of China (NAOC). The cluster has one management node, one login node and $60 \mathrm{com}$ puting nodes; each node has 1.28 TB DDR4-2133/2400 Ecc memory and two E5-2600v3/v4 series processors; software is Matlab R2012a glnxa64; hundreds of tasks can be processed in parallel. It takes about $16 \mathrm{~h}$ to process the observation data for a whole day with single task, from reading the GPS data to calculating Formula (8).

Compared with the DCB based grid method, the $B_{\text {arc }}{ }^{-}$ based PSGM depends on a prerequisite of intensive observation data. Only if an arc has at least one neighbor IPP to form Eq. (3), its $B_{\text {arc }}$ can appear in the matrix Eq. (5), otherwise its $B_{\text {arc }}$ may not be able to be solved out. In the GEONET observation data of 2014, the number of $B_{\mathrm{arc}} \mathrm{s}$ that cannot be solved accounts for about $8 \%$ of total available arcs.

As long as $B_{\text {arc }}$ is solved, the VTEC of the whole arc can be obtained by Formulas (1) and (2). If there is only one IPP in a $0.1^{\circ} \times 0.1^{\circ}$ grid, the VTEC of the IPP is considered as the VTEC of the grid. If there are multiple IPPs, the median VTEC of these IPPs is taken as the VTEC of the grid.

The observation data cannot guarantee to cover all the grids all the time as the grids are quite small. Taking the grid centered at $135^{\circ} \mathrm{E} 35^{\circ} \mathrm{N}$ (near Kobe) as an example, the VTEC available ratio is about $60 \%$, that is to say, around 1700 (60\% of 2880$)$ epochs in a day have VTEC measurements. The available ratio of grids $135^{\circ} \mathrm{E} 40^{\circ} \mathrm{N}$ and $135^{\circ} \mathrm{E} 30^{\circ} \mathrm{N}$ (above the ocean) decrease rapidly to around $15 \%$. This shows that the available ratio decreases rapidly due to observation density reduction.

\section{VTEC result}

In order to simplify the problem, the ionospheric shell height parameter is assumed to be a constant $h=400 \mathrm{~km}$ (Ma and Maruyama 2003). The GEONET observations of 2014 were fitted with PSGM. For comparison and analysis, we also calculated the VTEC based on the $1^{\circ} \times 1^{\circ}$ grid size, which is referred as Phase bias-based Big Grid Model (PBGM) VTEC. The algorithms of PBGM and PSGM are exactly the same except for the grid size. The IRI-VTEC and UNIBE-VTEC were introduced in some figures. Need to note that IRI-VTEC and UNIBE-VTEC can be considered as external reference and validation here rather than the object of criticism and evaluation because they are based on different backgrounds and environments.

Figure 1 displays the VTEC variation on 16 magnetically quiet $(\mathrm{Kp}<4)$ days of four seasons of 2014. The diurnal variation in PSGM-VTEC is consistent with UNIBE and IRI in general. The smoothness and continuity of PSGM are very good. The difference between PSGM and PBGM will not exceed 2 TECU in most cases, which shows the self-consistency and stability of the algorithm. The most obvious difference between the two is in Fig. 1b. The diurnal peaks of the PBGM result around noon are slightly higher than those of PSGM, and the fluctuation is more intense as well. This may indicate that PSGM is better than PBGM in accuracy.

Figure 2 displays the VTEC variation at different latitudes on 15-17 March 2014. By comparing PSGM-VTEC at $30^{\circ} \mathrm{N}$ in Fig. 2c for the three consecutive days, the PSGM-VTEC peaks of 15-17 March are 67.59 TECU, 98.37 TECU and 57.44 TECU. The PSGM-VTEC of 16 March is more than 30 TECU higher than that of 15 March and 17 March. This TEC enhancement gradually weakened with increasing latitude. The peak VTEC decreased rapidly from about $100 \mathrm{TECU}$ at $30^{\circ} \mathrm{N}$ to about $65 \mathrm{TECU}$ at $35^{\circ} \mathrm{N}$, that is to say, VTEC had a spatial gradient of up to $7 \mathrm{TECU} /{ }^{\circ}$. So in this case, the assumption of constant VTEC in a $1^{\circ} \times 1^{\circ}$ grid will undoubtedly lead to larger errors. Reflected in Fig. 2c, the VTEC curve of PBSM contains some burrs.

Figure 3 displays the VTEC seasonal variation at the grid point of $\left[135^{\circ} \mathrm{E}, 35^{\circ} \mathrm{N}\right]$ for daytime (04:00 UT, 13:00 JST) and nighttime (16:00 UT, 01:00 JST) of 364 days in 2014. In daytime, VTEC is the largest in spring, followed by autumn and winter, and smallest in summer. At nighttime, VTEC is the largest in summer, followed by spring, and then in autumn and winter. The trends of PSGMVTEC, PBGM-VTEC, IRI-VTEC and UNIBE-VTEC are similar.

The average difference between PSGM and PBGM is $5.04 \%$ (1.7 TECU) in daytime and $7.76 \%$ (0.88 TECU) 

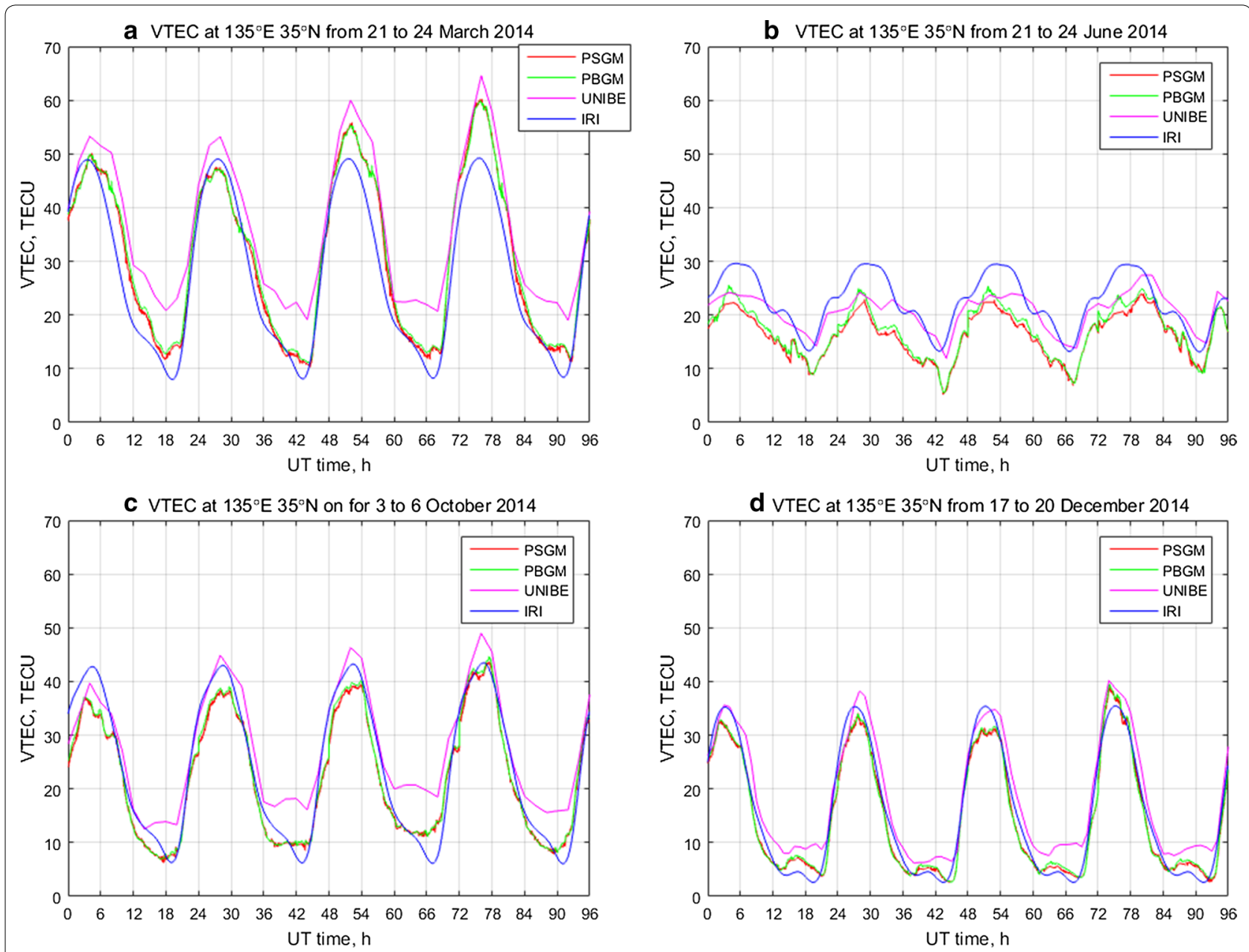

Fig. 1 VTEC diurnal variation of 2014 at $135^{\circ} \mathrm{E} 35^{\circ} \mathrm{N}$. a 21-24 March; b 21-24 June; c 3-6 October; d 17-20 December
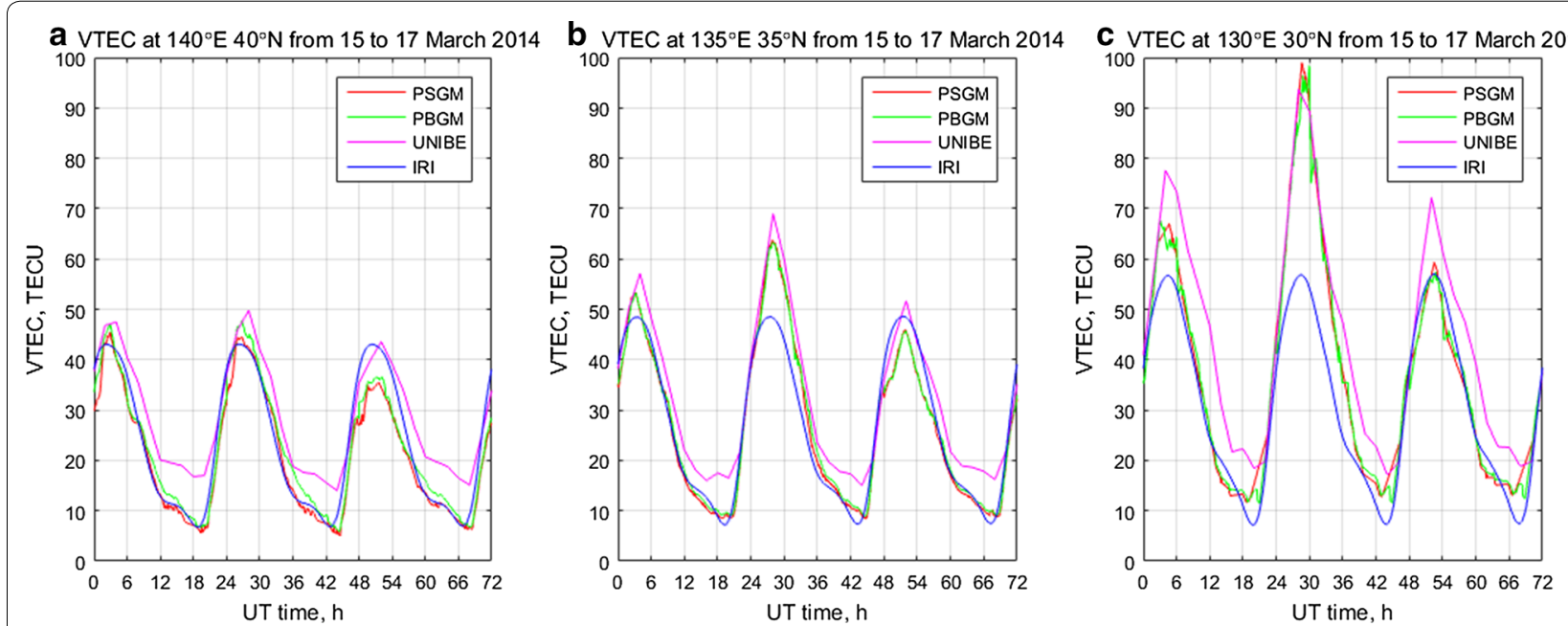

Fig. 2 VTEC variation of $15-17$ June 2014 at different latitudes: a $130^{\circ} \mathrm{E} 30^{\circ} \mathrm{N}$; b $135^{\circ} \mathrm{E} 35^{\circ} \mathrm{N}$; c $140^{\circ} \mathrm{E} 40^{\circ} \mathrm{N}$ 


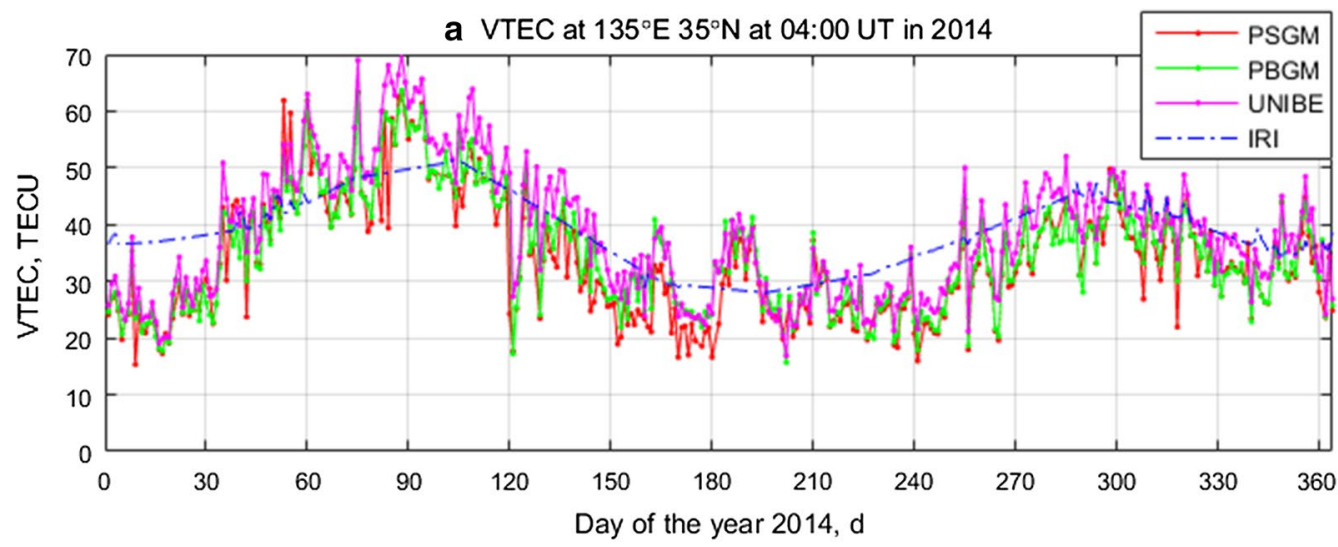

b VTEC at $135^{\circ} \mathrm{E} 35^{\circ} \mathrm{N}$ at $16: 00 \mathrm{UT}$ in 2014

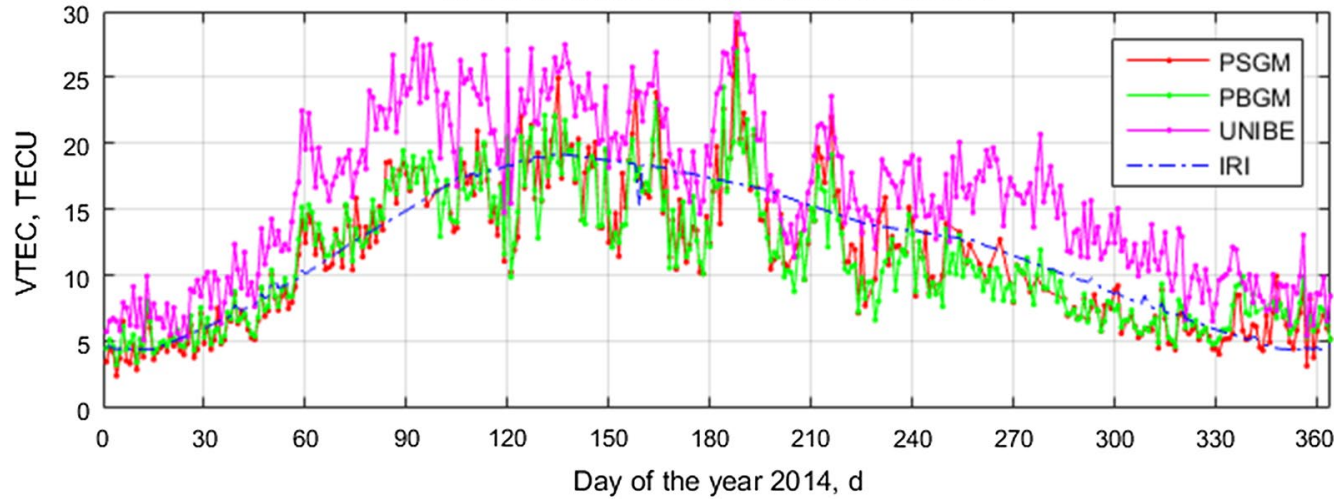

c F10.7 index in 2014

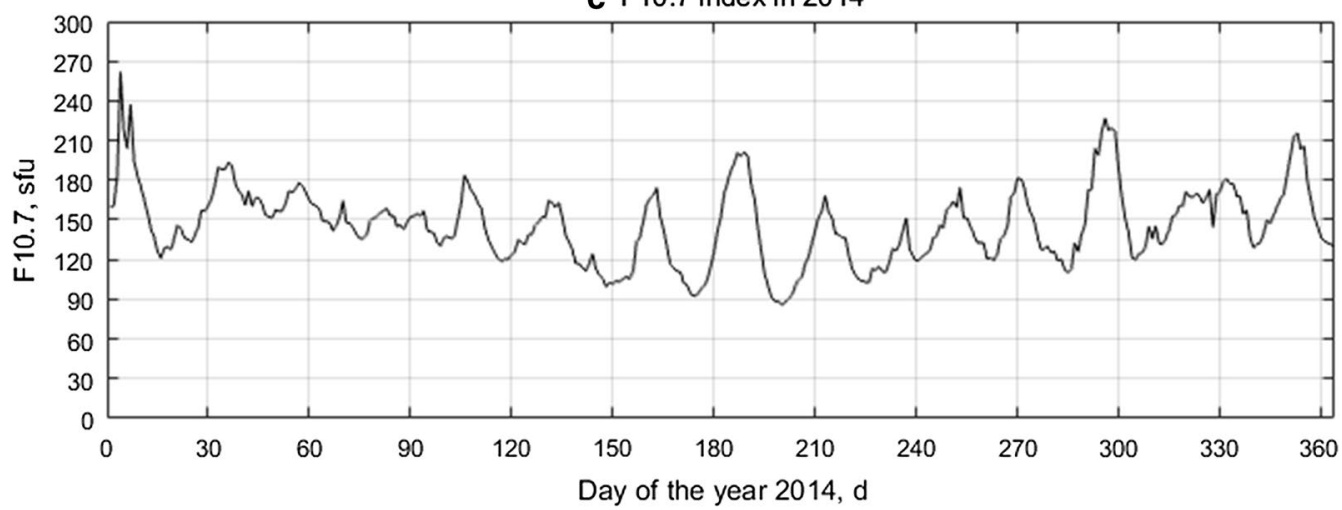

Fig. 3 VTEC seasonal variation of 2014 at $135^{\circ} \mathrm{E} 35^{\circ} \mathrm{N}$. a 04:00 UT; b 16:00 UT; c F10.7 Index

in nighttime. In Fig. 3b, the nighttime UNIBE-VTEC is generally larger than PSGM-VTEC, which may be caused by these reasons: (1) the shell height parameter is $450 \mathrm{~km}$ in UNIBE, VTEC may be slightly overestimated than $400 \mathrm{~km}$ (Wang et al. 2016); (2) the UNIBEVTEC is provided every $2.5^{\circ}$ of latitudes, lower latitude coverage $\left(33.75^{\circ} \mathrm{N}\right.$ to $\left.35^{\circ} \mathrm{N}\right)$ may enlarge the VTEC estimation at $35^{\circ} \mathrm{N}$; (3) the assumption that $\mathrm{DCB}$ in one day is unchanged may overestimate nighttime VTEC compared with the assumption that phase bias of an arc is unchanged.

The curve of PSGM-VTEC is quite consistent with that of F10.7 from April to August from Fig. 3. The correlation coefficient of the PSGM-VTEC and the F10.7 is 0.63 in nighttime during April to August while it is only -0.22 for the whole year 2014. In daytime, the correlation coefficient is 0.52 during April to August and 0.30 for the whole year. It shows that solar radiation is an important 
factor affecting absolute VTEC especially in summer night.

Figure 4 displays the two-dimensional VTEC maps at each hour from 10:00 UT to 19:00 UT of 11 July 2014. The map coverage is between $125^{\circ} \mathrm{E}-150^{\circ} \mathrm{E}$ and $25^{\circ} \mathrm{N}-50^{\circ} \mathrm{N}$. Every 2 TECU is displayed as a color level. VTEC contours are clearly exhibited in Fig. 4a. VTEC decreased gradually with the increase of latitude. From 12:00 UT to 15:00 UT, a series of northwest-southeast (NW-SE) wave stripes were formed. These structures had wavelengths of several hundred kilometers, propagated to southwest at $\sim 150 \mathrm{~m} / \mathrm{s}$ and recovered gradually after 16:00 UT, which is believed to be a typical nighttime medium-scale traveling ionospheric disturbances (MSTID) (Tsugawa et al. 2007). The example in Fig. 4 shows that PSGM can obtain VTEC measurements for the whole Japan region, and PSGM can at least reflect the structure and evolution of ionospheric disturbances that greater than 2.0 TECU level.

\section{Cross-validation}

Though there are some comparisons between PSGM and PBGM in "VTEC result" section, they cannot be used as the exact evidence to explain the accuracy of the method as they all derived from the same observation data. To further illustrate the accuracy of PSGM, all GEONET stations are randomly divided into two groups for cross-validation. The stations of the two groups are completely different. The PSGM-VTEC results of these two groups are named GROUPA-VTEC and GROUPBVTEC, respectively. Figure 5 shows the comparison of fitting results on 4 example days, the average difference at $135^{\circ} \mathrm{E} 35^{\circ} \mathrm{N}$ between GROUPA-VTEC and GROUPBVTEC is only 0.36 TECU. If all the grids with both sets of VTEC results are counted in, the average VTEC difference between the two groups is 0.46 TECU and only 1.92\% grid VTEC differences are higher than 2.0 TECU. Cross-validation further proves the accuracy of PSGM algorithm. It should be noted that since the number of

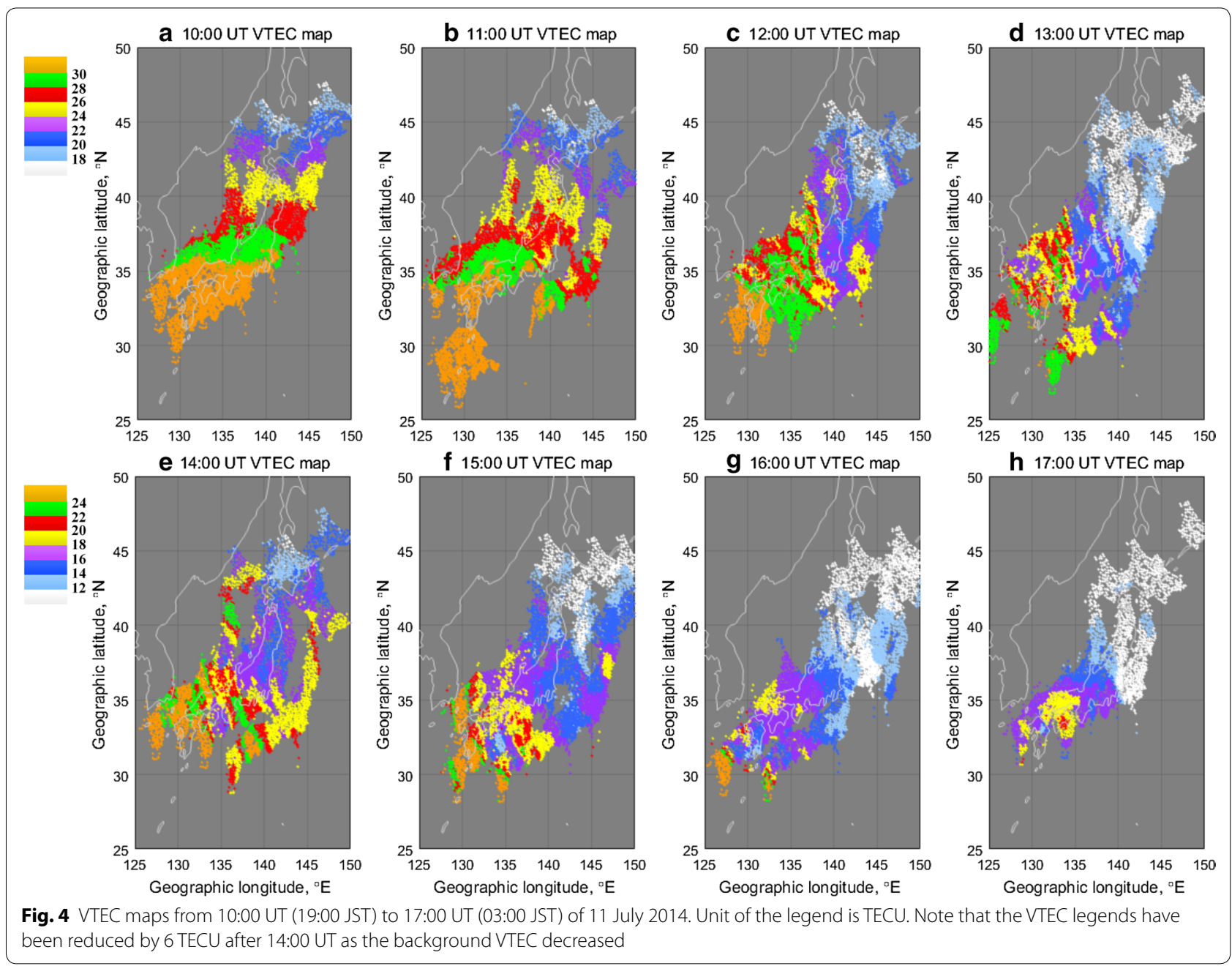



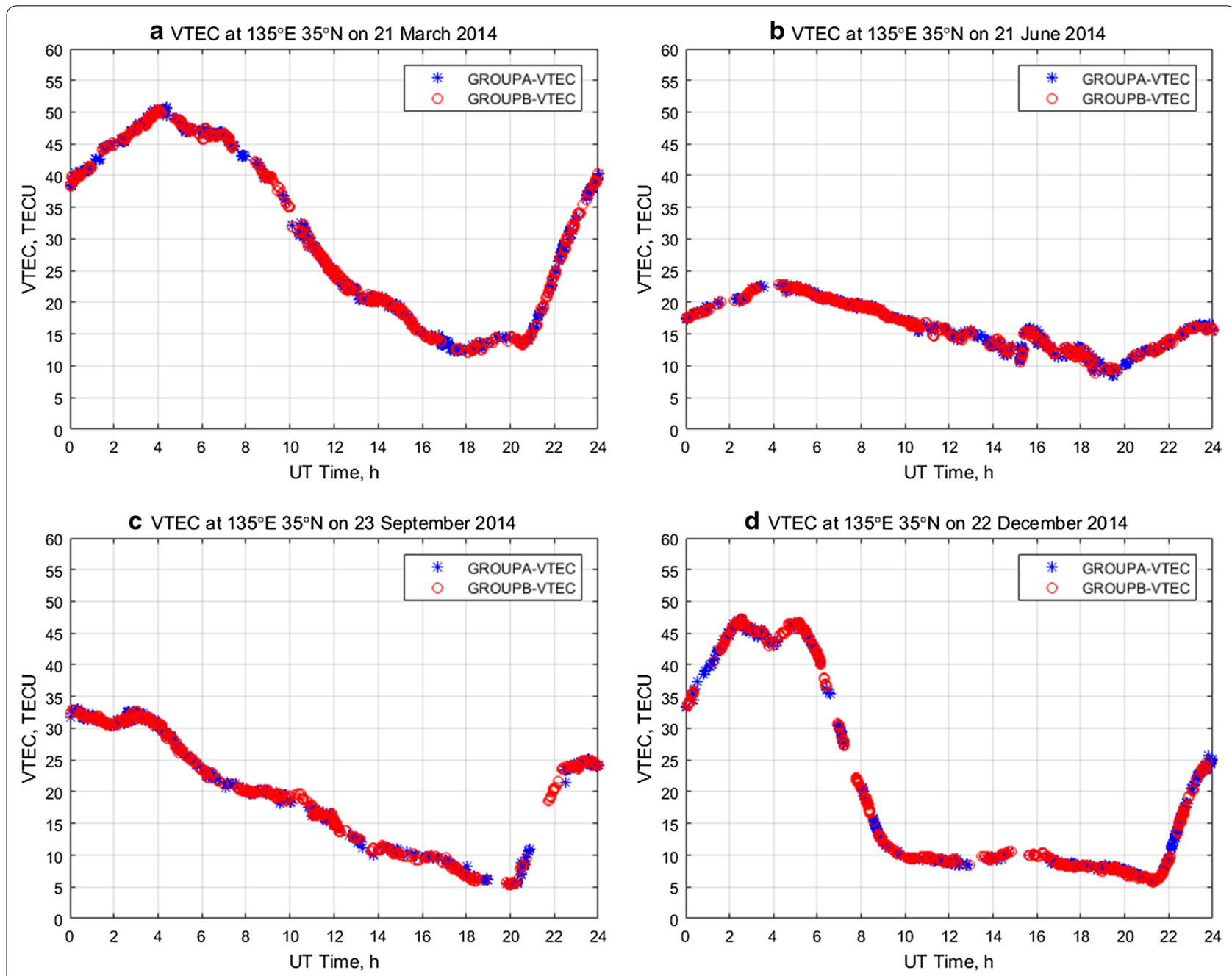

Fig. 5 PSGM-VTEC cross-validation. a 21 March; b 21 June; c 23 September; d 22 December

stations of each group is reduced by half, the number of available IPP neighbor pairs is also greatly reduced, so the fitting accuracy of total stations should be higher than that of any group.

\section{RMSE result}

Parameter RMSE in Formula (8) can reflect how well the data is fitted. The RMSE level is the possible difference between the two measurements in the same grid. Figure 6 shows the RMSE result in 2014. On the whole, PSGM-RMSE is the largest in summer and smallest in winter. The average PSGM-RMSE of 364 days is 0.40 TECU. The maximum PSGM-RMSE is 0.73 TECU on 13 May and the minimum RMSE is 0.26 TECU on 21 February. This means that PSGM is appropriate to be applied to the study of ionospheric disturbances at 1.0 TECU level theoretically.
As a contrast, the average PBGM RMSE of 364 days is 0.64 TECU. The maximum PBGM RMSE is 1.12 TECU on 16 March and the minimum RMSE is 0.39 TECU on 18 January. PSGM-RMSE is about $37 \%$ less than PBGM RMSE. On the other hand, the biggest season of PBGM RMSE has changed to spring, which is caused by the larger TEC spatial gradient in spring. The mean value of the rate of TEC index (ROTI) (Pi et al. 1997) of all observation in a day is introduced as the measurement of ionospheric disturbing level. Figure $6 \mathrm{~b}$ examined the relationship between PSGM-RMSE and mean ROTI. It can be seen that PSGM-RMSE increases with the increase of mean ROTI. The correlation between ROTI and PSGM-RMSE is a clue that the accuracy of PSGM can be affected by ionospheric disturbances.

PSGM can also be applied to a short period rather than an entire day. Figure 7 shows the PSGM-RMSE obtained 

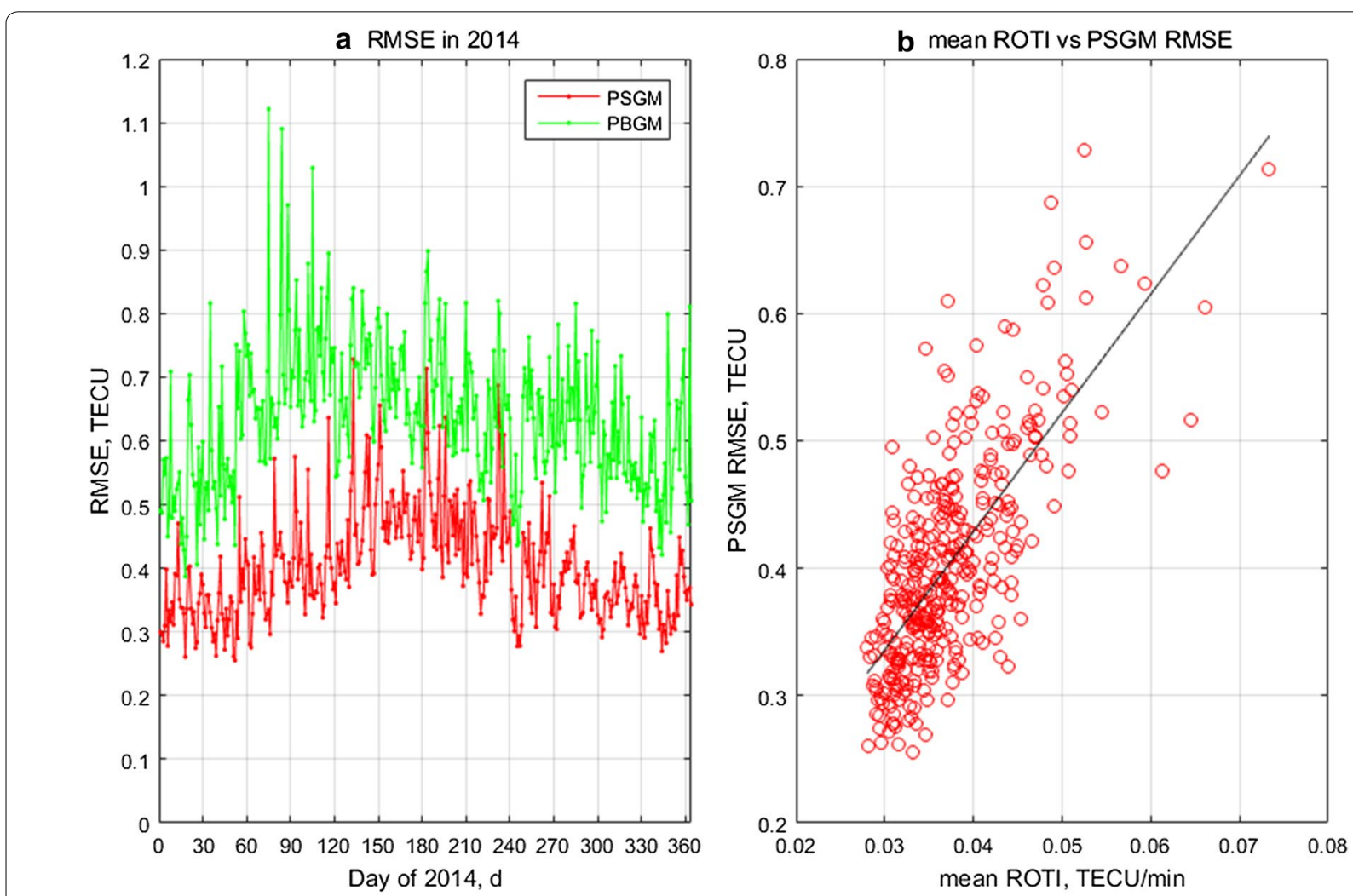

Fig. 6 RMSE of PSGM and PBGM. a RMSE for 364 days in 2014; b a scatter plot for comparison of the PSGM-RMSE to mean ROTI for 364 days in 2014 Slope of the fitted straight line is 9.3

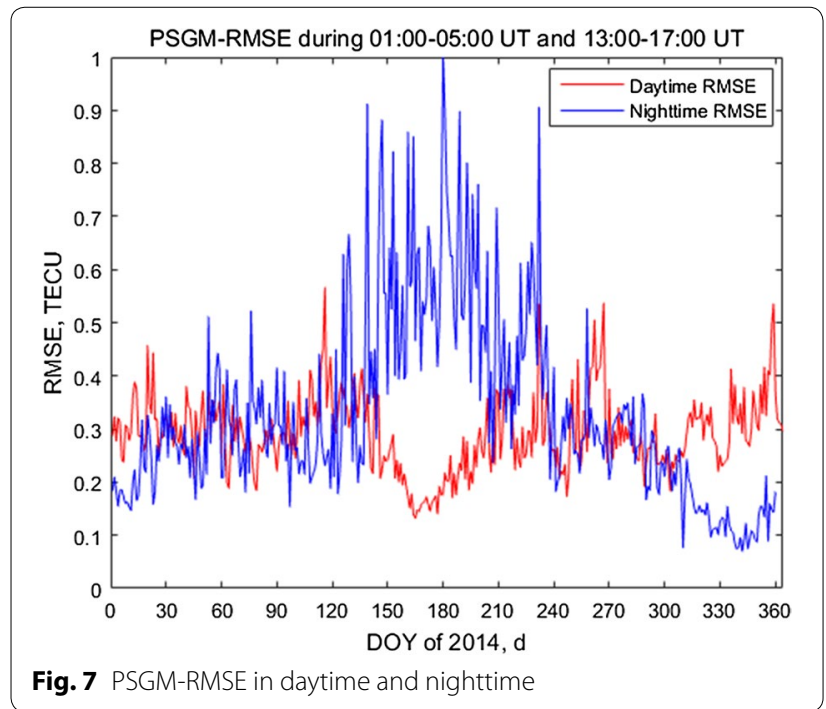

by fitting the observation data of $4 \mathrm{~h}$ in the daytime and the nighttime, respectively. In summer, the daytime PSGM-RMSE is smallest, but the nighttime PSGMRMSE is largest. And in winter, PSGM-RMSE increased in daytime and decreased at night. It is interesting that the relationship between the daytime RMSE and nighttime RMSE is opposite in winter and summer. On the whole, though the daytime VTEC is much larger, the average RMSE at night ( 0.33 TECU) is slightly larger than that during the day (0.29 TECU), which is also consistent with the objective reality that more ionospheric disturbances happened at night.

\section{Shell height parameter optimization}

At the beginning of "VTEC result" section, we assume that the height of ionospheric shell is fixed at $400 \mathrm{~km}$ to simplify the calculation. But actually, the height of the ionospheric shell always changes with time and place (Wang et al. 2016; Lu et al. 2017). For PSGM, the change of shell height parameter is easier to map IPPs to different grids under different satellite elevation than PBGM. In turn, this feature can also be used to optimize the ionospheric shell height parameter.

When the shell height parameter changes, not only the grid mapping of the IPP will change, but also the zenith angle $\chi$, so PSGM-RMSE usually does not change smoothly with the shell height parameter. In addition, due to the narrow station distribution of GEONET, when the shell height parameter is too large, the number of 
neighbor IPP pairs composed of different satellites will be greatly reduced, and the PSGM-RMSE will always tend to decrease as shell height parameter increases. Even though, at most of the time, we can still find a shell height parameter between 250 and $600 \mathrm{~km}$ which can make PSGM-RMSE minimum. Figure 8 shows the relationship between RMSE and shell height parameter of the 6 example days 01:00-05:00 UT in 2014. We performed calculation every $10 \mathrm{~km}$ of $250-600 \mathrm{~km}$ according to the shell height parameter and keep all other conditions unchanged. The shell height parameter of minimum PSGM-RMSE in these 6 days is $340 \mathrm{~km}, 390 \mathrm{~km}, 390 \mathrm{~km}$, $450 \mathrm{~km}, 440 \mathrm{~km}$ and $360 \mathrm{~km}$. The reason why we choose the observation data of $4 \mathrm{~h}$ at noon rather than the entire day is that the ionosphere height changes the least and the TEC value is the highest during this period.

Figure 9 shows the variation of the optimum height $(\mathrm{OH})$ parameters estimated by the minimum RMSE method during 01:00-05:00 in 2014. The overall trend is the lowest in winter and the highest in summer, which is consistent with the result estimated by Zhao and Zhou (2018) using single station DCB method. The OH in summer is often over $500 \mathrm{~km}$; while in winter it is often as low as $300 \mathrm{~km}$ but never less than $250 \mathrm{~km}$. The parabola in the figure is the trend of the shell height parameter variation fitted by polynomial, which is consistent with the trend of hmF2 in IRI. PSGM-RMSE under the OH parameter is about $4.4 \%$ less than that of $400 \mathrm{~km}$. It shows that there is a potential to further improve the fitting accuracy if the variable shell height parameter is used instead of the constant shell height parameter.

\section{Summary}

The accuracy of VTEC estimation using dual-frequency GNSS system is a very meaningful research topic. In this paper, the RMSE of VTEC fitting residuals is analyzed by using the GEONET observation and the PSGM. The PSGM is based on two assumptions. The first assumption
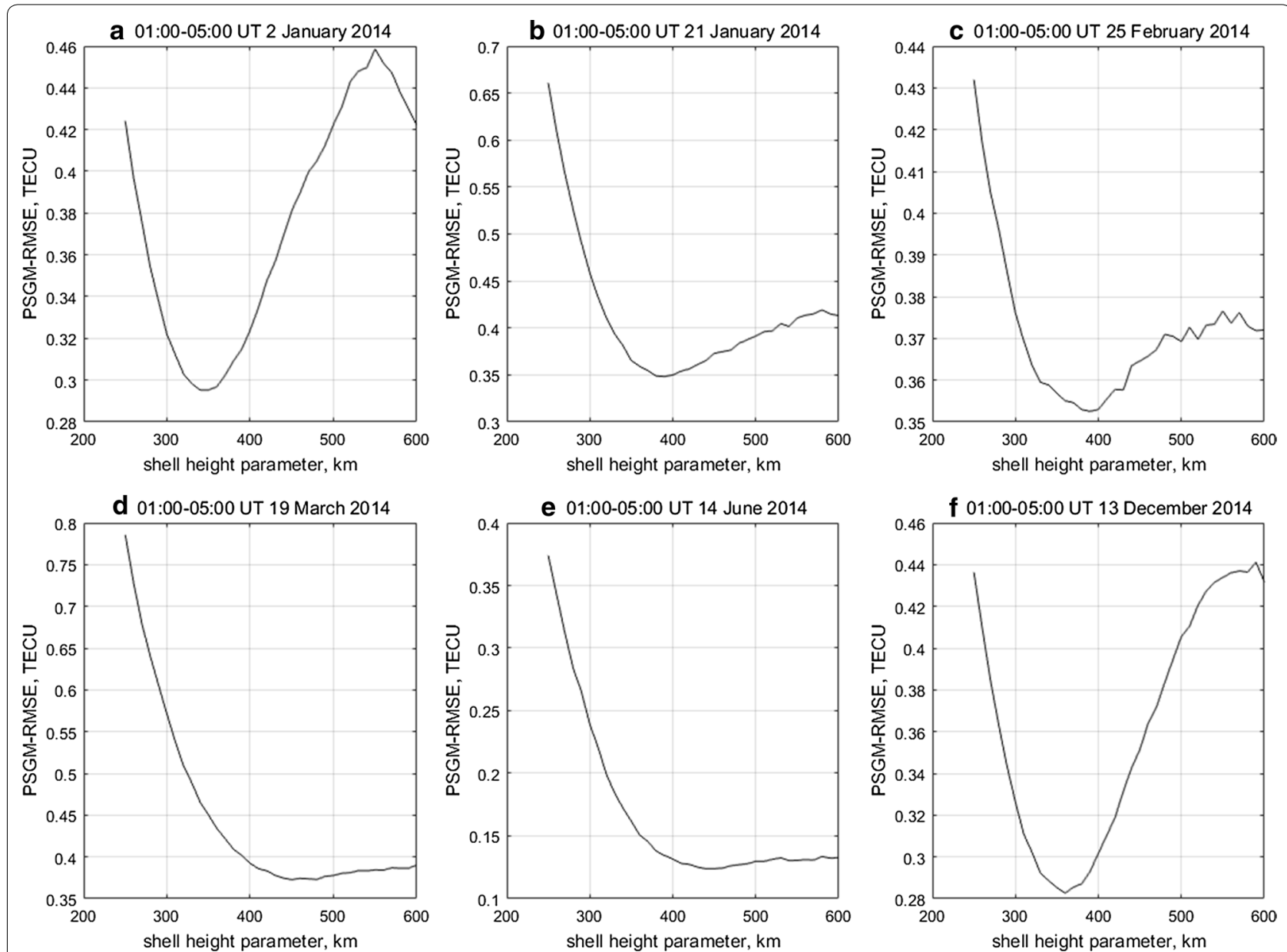

Fig. 86 example days PSGM-RMSE vs shell height parameter during daytime. a 2 January; b 21 January; c 25 February; d 19 March; e 14 June; f 13 December 


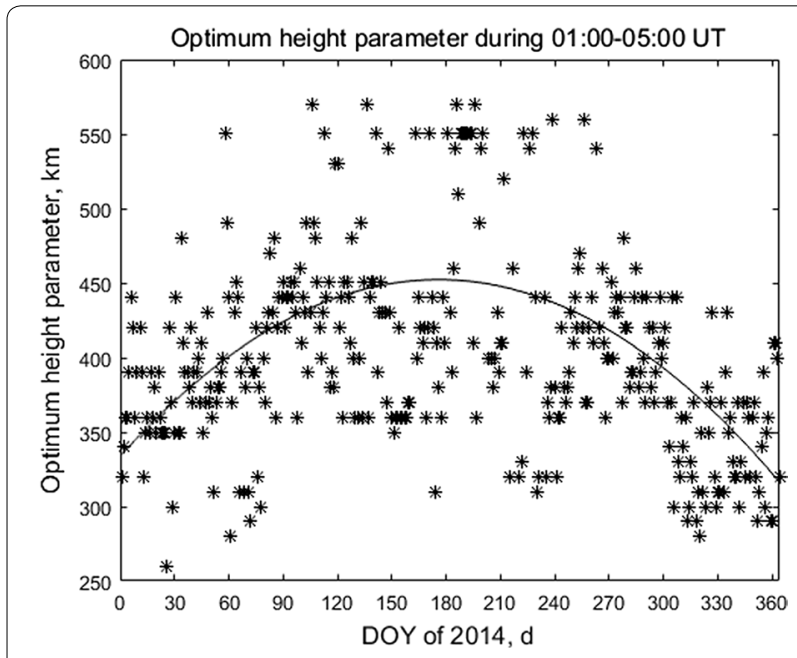

Fig. 9 Optimum shell height parameter during 01:00 UT to 05:00 UT in 2014

that the integer ambiguities and hardware delay biases of dual-frequency GPS phase observation are invariant within the same phase connected arc can help to get rid of the dependence on low-precision pseudorange measurements. The second assumption that the VTEC is identical in the same $0.1^{\circ} \times 0.1^{\circ}$ grid can minimize the prerequisite for spatio-temporal smoothing.

The trends of PSGM, PBGM, UNIBE and IRI are consistent in both diurnal and seasonal variations. PSGM can reduce the impact of large TEC spatial gradient. The PSGM-VTEC can be used to detect some ionosphere phenomena such as VTEC enhancement and MSTID.

The cross-validation error is about $0.46 \mathrm{TECU}$ in average. The PSGM-RMSE in summer is larger than that in winter and increases with the increase of mean ROTI. It is found that under the simple isotropic fixed height ionospheric shell model, the PSGM-RMSE is as low as 0.40 TECU in average in the year of 2014. This progress will refresh the understanding of the upper limit of the fitting accuracy for thin shell model. The trend of PSGM-RMSE is different in daytime and nighttime. In daytime, PSGMRMSE is the largest in winter and the smallest in summer, and it is the opposite at night.

PSGM excludes most error sources and minimizes the impact of spatial variation, so that the difference between the assumed shell height and the actual shell height can be reflected in the change of RMSE. By comparing the PSGM-RMSE corresponding to different shell height parameters, the $\mathrm{OH}$ parameter that can minimize the RMSE can be found. In 2014, the $\mathrm{OH}$ in the daytime is the lowest in winter and the highest in summer.
Abbreviations

GNSS: Global Navigation Satellite System; GPS: Global Positioning System; DCB differential code bias; GEONET: GNSS Earth Observation Network of Japan; IPP: ionospheric piercing point; IRI: International Reference lonosphere; IGS: International GNSS Service; JST: Japan standard time; MSTID: medium-scale traveling ionospheric disturbances; NAOC: National Astronomical Observatories of China; NOAA: National Oceanic And Atmospheric Administration: NW-SE: northwest-southeast; OH: optimum height; PBGM: Phase bias-based Big Grid Model; PSGM: Phase bias-based Small Grid Model; RMSE: root mean square error; ROTI: rate of TEC index; TEC: total electron content; VTEC: vertical total electron content; UNIBE: University of Bern; UT: Universal time.

\section{Acknowledgements}

The author is grateful to the reviewers for their valuable suggestions. The computing platform is at Information and Computing Center of NAOC. IRI2016 model is provided by http://irimodel.org/. The F10.7 data are provided by NOAA. The UNIBE ionosphere products are provided by IGS. This work is conducted with the support of the National Natural Science Foundation of China (Grant Nos. 11473045, 11873064 and 11503040) and the National Key Research Program of China "Collaborative Precision Positioning Project" (Nos. 2016YFB0501903 and 2016YFB0501904)

\section{Authors' contributions}

QL: modeling; algorithm design; coding and debugging; manuscript writing; figures plotting. GM: guidance; modeling; proposing idea; framework design; manuscript revising; figures analyzing. TM: guidance; data acquisition; manuscript revising. JL: key issues discussion; manuscript revising. QW: data acquisition; key issues discussion. XW: data acquisition; key issues discussion. JF: key issues discussion. JZ: key issues discussion. All authors read and approved the final manuscript.

\section{Funding}

This work is conducted with the support of the National Natural Science Foundation of China (Grant Nos. 11473045, 11873064 and 11503040) and the National Key Research Program of China "Collaborative Precision Positioning Project" (Nos. 2016YFB0501903 and 2016YFB0501904).

\section{Availability of data and materials}

The GEONET data were provided by Geospatial Information Authority of Japan: http://datahouse1.gsi.go.jp/terras/terras_english.html

IRI2016 model is provided by http://irimodel.org/.

The F10.7 data is provided by NOAA: ftp://ftp.ngdc.noaa.gov/STP/space -weather/solar-data/solar-features/solar-radio/noontime-flux/penticton/penti cton_observed/listings.

UNIBE ionosphere products is provided by IGS: ftp://igs.ensg.ign.fr/pub/igs/ products/ionosphere.

Ethics approval and consent to participate

Not applicable.

Consent for publication

Not applicable.

\section{Competing interests}

The authors declare that they have no competing interests.

\section{Author details}

${ }^{1}$ National Astronomical Observatories, Chinese Academy of Sciences, Beijing, China. ${ }^{2}$ University of Chinese Academy of Sciences, Beijing, China. ${ }^{3}$ National Institute of Information and Communications Technology, Koganei, Tokyo, Japan.

Received: 31 August 2019 Accepted: 18 January 2020

Published online: 05 February 2020

References

Arikan F, Nayir H, Sezen U, Arikan O (2008) Estimation of single station interfrequency station bias using GPS-TEC. Radio Sci 43(4):RS4004. https://doi. org/10.1029/2007RS003785 
Bilitza D, Altadill D, Truhlik V, Shubin V, Galkin I, Reinisch B, Huang X (2017) International Reference lonosphere 2016: from ionospheric climate to real-time weather predictions. Space Weather 15(2):418-429. https://doi. org/10.1002/2016SW001593

Blewitt G (1990) An automatic editing algorithm for gps data. Geophys Res Lett 17(3):199-202. https://doi.org/10.1029/GL017i003p00199

Choi B-K, Park J-U, Roh KM, Lee S-J (2013) Comparison of GPS receiver DCB estimation methods using a GPS network. Earth Planets Space 65(7):707-711

Coco DS, Coker C, Dahlke SR, Clynch JR (1991) Variability of GPS satellite differential group delay biases. IEEE Trans Aerosp Electron Syst 27(6):931-938. https://doi.org/10.1109/7.104264

Coster A, Williams J, Weatherwax A, Rideout W, Herne D (2013) Accuracy of GPS total electron content: GPS station bias temperature dependence. Radio Sci 48(2):190-196. https://doi.org/10.1002/rds.20011

Han D, Kim D, Kee C (2018) Improving performance of GPS satellite DCB estimation for regional GPS networks using long-term stability. GPS Solut 22(1):13. https://doi.org/10.1007/s10291-017-0669-7

Hernández-Pajares M, Juan JM, Sanz J (1999) New approaches in global ionospheric determination using ground GPS data. J Atmos Solar Terr Phys 61(16):1237-1247. https://doi.org/10.1016/S1364-6826(99)00054-1

Lanyi GE, Roth T (1988) A comparison of mapped and measured total ionospheric electron content using global positioning system and beacon satellite observations. Radio Sci 23(4):483-492. https://doi.org/10.1029/ RS023i004p00483

Li LX, Zhang DH, Zhang SR, Coster AJ, Hao YQ, Xiao Z (2015) Influences of the day-night differences of ionospheric variability on the estimation of GPS differential code bias. Radio Sci 50(4):339-353. https://doi. org/10.1002/2014RS005565

Li Q, Ma G, Lu W, Wan Q, Fan J, Wang X, Li J, Li C (2018) A method of estimating GPS instrumental biases with a convolution algorithm. Adv Space Res 61(6):1387-1397. https://doi.org/10.1016/j.asr.2017.11.034

Lu W, Ma G, Wang X, Wan Q, Li J (2017) Evaluation of ionospheric height assumption for single station GPS-TEC derivation. Adv Space Res 60(2):286-294. https://doi.org/10.1016/j.asr.2017.01.019

Ma G, Maruyama T (2003) Derivation of TEC and estimation of instrumental biases from GEONET in Japan. Ann Geophys 21(10):2083-2093 (hal-00317176)

Ma XF, Maruyama T, Ma G, Takeda T (2005) Determination of GPS station differential biases by neural network parameter estimation method. Radio Sci 40(1):RS1002. https://doi.org/10.1029/2004RS003072

Mannucci AJ, Wilson BD, Yuan DN, Ho CH, Lindqwister UJ, Runge TF (1998) A global mapping technique for GPS derived ionospheric electron content measurements. Radio Sci 33(3):565-582. https://doi.org/10.1029/97RS0 2707

Montenbruck O, Hauschild A, Steigenberger P (2014) Differential code bias estimation using multi-GNSS observations and global ionosphere maps. Navigation 61(3):191-201. https://doi.org/10.1002/navi.64
Otsuka Y, Ogawa T, Saito A, Tsugawa T, Fukao S, Miyazaki S (2002) A new technique for mapping of total electron content using GPS network in Japan. Earth Planets Space 54(1):63-70. https://doi.org/10.1186/BF03352422

Perez RO (2019) Using TensorFlow-based Neural Network to estimate GNSS single frequency ionospheric delay (IONONet). Adv Space Res 63(5):1607-1618. https://doi.org/10.1016/j.asr.2018.11.011

Pi X, Mannucci AJ, Lindqwister UJ, Ho CM (1997) Monitoring of global ionospheric irregularities using the worldwide GPS network. Geophys Res Lett 24(18):2283-2286

Sardon E, Rius A, Zarraoa N (1994) Estimation of the transmitter and station differential biases and the ionospheric total electron content from Global Positioning System observations. Radio Sci 29(03):577-586. https://doi. org/10.1029/94RS00449

Sarma AD, Rao GS, Rao PVDS, Ramalingam K (2008) Gps satellite and station instrumental biases estimation using svd algorithm. IEEE Trans Aerosp Electron Syst 44(4):1560-1566. https://doi.org/10.1109/TAES.2008.46677 31

Schaer S, Beutler G, Rothacher M, Springer TA (1996). Daily global ionosphere maps based on GPS carrier phase data routinely produced by the CODE Analysis Center. In: Proceedings of the IGS Analysis Center Workshop 1996

Tsugawa T, Kotake N, Otsuka Y, Saito A (2007) Medium-scale traveling ionospheric disturbances observed by GPS station network in Japan: a short review. GPS Solut 11(2):139-144. https://doi.org/10.1007/s1029 1-006-0045-5

Wang XL, Wan QT, Ma GY, Li JH, Fan JT (2016) The influence of ionospheric thin shell height on TEC retrieval from GPS observation. Res Astron Astrophys 16(7):116. https://doi.org/10.1088/1674-4527/16/7/116/meta

Wilson BD, Mannucci AJ, Edwards CD (1995) Subdaily northern hemisphere ionospheric maps using an extensive network of GPS stations. Radio Sci 30(3):639-648. https://doi.org/10.1029/94RS03186

Zhang W, Zhang DH, Xiao Z (2009) The influence of geomagnetic storms on the estimation of GPS instrumental biases. Ann Geophys 27(4):16131623. https://doi.org/10.5194/angeo-27-1613-2009

Zhang DH, Zhang W, Li Q, Shi LQ, Hao YQ, Xiao Z (2010) Accuracy analysis of the GPS instrumental bias estimated from observations in middle and low latitudes. Ann Geophys 28(8):1571-1580. https://doi.org/10.5194/ angeo-28-1571-2010

Zhao J, Zhou C (2018) On the optimal height of ionospheric shell for singlesite TEC estimation. GPS Solut 22(2):48. https://doi.org/10.1007/s1029 $1-018-0715-0$

\section{Publisher's Note}

Springer Nature remains neutral with regard to jurisdictional claims in published maps and institutional affiliations.

\section{Submit your manuscript to a SpringerOpen ${ }^{\odot}$ journal and benefit from:}

- Convenient online submission

- Rigorous peer review

- Open access: articles freely available online

- High visibility within the field

Retaining the copyright to your article

Submit your next manuscript at springeropen.com 